\title{
Best allogeneic transplantation approach for AML patients in first CR: is delayed unrelated donor bone marrow transplantation better than immediate unrelated cord blood transplantation?
}

\author{
Frédéric Baron ${ }^{1,2}$, Arnon Nagler $3,4,5,6$ \\ ${ }^{1}$ Department of Medicine, Division of Hematology, University and CHU of Liège, Liège, Belgium; ${ }^{2}$ Giga-I3, Section of Hematology, University of \\ Liège, Liège, Belgium; ${ }^{3}$ Division of Hematology and Bone Marrow Transplantation, The Chaim Sheba Medical Center, Tel-Hashomer, Ramat-Gan, \\ Israel; ${ }^{4}$ EBMT Paris Office, Hospital Saint Antoine, Paris, France; ${ }^{5}$ Université Pierre et Marie Curie, Paris, France; ${ }^{6}$ Tel Aviv University (TAU), Tel \\ Aviv, Israel
}

Allogeneic hematopoietic cell transplantation (allo-HCT) with an HLA-identical sibling donor is the best postremission treatment for patients with non-good-risk acute myeloid leukemia (AML) in first complete remission (CR) (1). For patients who lack an HLA-identical sibling donor, transplantation with an $8 / 8$ or a $7 / 8$ HLA-matched unrelated donor, an HLA-haploidentical donor, or an unrelated umbilical cord blood (UCB) are considered as valid alternatives (1).

Potential advantages of UCBT in comparison with bone marrow (BMT) or peripheral blood stem cell (PBSCT) transplantation include more rapid availability and, because UCB is relatively deficient in mature $\mathrm{T}$ cells, a low incidence of chronic GVHD despite a greater degree of accepted HLA mismatching (2). Disadvantages of UCB include slower engraftment and increased risks of graft failure, both due at least in part to the low number of cells contained in one UCB unit (2). A large study by the Center for International Blood and Marrow Transplant Research and the Acute Leukemia Working Party of the European Group for Blood and Marrow transplantation assessed the impact of graft source (unrelated PBSC, unrelated BMT or UCB) on transplantation outcomes in a cohort of 1525 patients with acute leukemia transplanted between 2002 and 2006 (3). Main observations were that engraftment kinetics were slower in UCB recipient than in BMT or PBSCT recipients. Incidences of grade IIIV acute GVHD were lower after UCBT than after PBSCT but similar in UCB and BMT recipients, while incidences of chronic GVHD were lower in UCB recipients than in patients transplanted with other stem cell sources. Finally, although non-relapse mortality (NRM) was higher in UCB patients than in those receiving HLA-matched BM or PBSC,

Correspondence Frédéric Baron, Department of Medicine, Division of Hematology, University and $\mathrm{CHU}$ of Liège, Liège, Belgium. Tel: +32 436672 01; Fax: +32 436688 55; e-mail: f.baron@ulg.ac.be and

Arnon Nagler, Division of Hematology and Bone Marrow Transplantation, The Chaim Sheba Medical Center, Tel-Hashomer, Ramat-Gan, Israel. Tel: 9723 5305830; Fax: 9723 5304792.5; e-mail: arnon.nagler@ sheba.health.gov.il leukemia-free survival (LFS) was similar with each stem cell source.

A possible bias of registry studies comparing UCBT with BMT or PBSCT is that only patients who undergo allo-HCT are taken into consideration. This might create an artificial disadvantage for UCBT (which is rapidly available), while patients scheduled for an unrelated allo-HCT can relapse while waiting for an unrelated donor to be found. In the current issue of the journal, Yanada et al. report the results of a study that used a decision analysis technique with the aim of comparing unrelated BMT with UCBT in patients with nongood-risk AML in first CR. Decision analysis is an increasingly used statistical technique that allows estimation of outcome given multiple variations of initial conditions and assumptions. The best treatment option is then determined based on the area under the survival curves, thus taking into consideration the impact of each early and late evens (4). The study population included a cohort of 907 patients from the Japan Adult Leukemia Study Group with intermediateor high-/very high-risk AML in CR1 given chemotherapy only, and a cohort of 752 patients from Japan Society for Hematopoietic Cell Transplantation transplanted with bone marrows from an 8/8 $(n=331)$ or a $7 / 8(n=169)$ HLAmatched unrelated donors or with UCB $(n=252)$. All patients were aged 16 to 54 years at diagnosis, and patients given in vivo or ex vivo T-cell depletion of the graft were excluded. Further, only patients transplanted in CR1 from 2 to 6 months after diagnosis for UCBT and from 4 to 10 months after diagnosis for unrelated BMT were included. Main observations were that, in the baseline analyses, 5-year OS was $60 \%$ for unrelated BMT recipients (results were similar in the group of patients given 8/8 HLA-matched BMT or $7 / 8$ HLA-matched BMT) versus $52 \%$ for UCB recipients. In sensitivity analyses, superiority of unrelated BMT over UCBT was consistently observed when the time from CR1 to unrelated BMT varied from 3 to 9 months, and the same held true for 7/8 HLA-matched unrelated BMT versus UCB. Given the lower incidence of chronic GVHD associated with UCBT, the authors performed additional analyses adjusted for quality of live (QOL) taking into 
consideration being alive with or without chronic GVHD at 5 years. In these analyses, a value of 98 was assigned to patients alive without chronic GVHD at 5 years, a value of 0 was assigned to death patients, and a value of 70 (with a wide plausible range from 0 to 98 ) assigned to patients alive with active chronic GVHD at 5 years. Notably, the expected OS rate for unrelated BMT remained higher than for UCBT when the utility of being alive with active GVHD was varied between 0 and 98 . The authors concluded that, based on their results, $8 / 8$ or $7 / 8 \mathrm{UBMT}$ is a better transplantation approach than UCBT even after taking into consideration the time required for donor coordination.

This study adds important information on the best allogeneic transplantation strategies for patients with AML in CR1 who lack an HLA-identical sibling donor, and the authors should be congratulated for having conducted thorough statistical analyses taking into consideration the different times requested to perform unrelated BMT or UCBT. However, this study has also some limitations such as the relative small number of patients included in the UCB group $(n=252)$, the lack of data on reason for performing UCB or unrelated BMT, the lack of data on genetic AML marker (which has been demonstrated to have an important impact on post-transplantation outcomes (5)), and the inclusion in the UCBT group patients who were given $<2.5 \times 10 \mathrm{E} 7$ $\mathrm{TNC} / \mathrm{kg}$ that might be considered as inadequate. Thus, more studies are needed before it can be definitively concluded that BMT with a 7/8 HLA-matched unrelated donor results in better outcome than UCBT.

\section{Disclosures}

The authors have no conflict of interest.

\section{Acknowledgements}

FB is Senior Research Associate at the National Fund for Scientific Research (FNRS) Belgium.

\section{References}

1. Cornelissen JJ, Blaise D. Hematopoietic stem cell transplantation for patients with AML in first complete remission. Blood 2016;127:62-70.

2. Baron F, Ruggeri A, Nagler A. Methods of ex vivo expansion of human cord blood cells: challenges, successes and clinical implications. Exp Rev Hematol 2016;9:297-314.

3. Eapen M, Rocha V, Sanz G, et al. Effect of graft source on unrelated donor haemopoietic stem-cell transplantation in adults with acute leukaemia: a retrospective analysis. Lancet Oncol 2010;11:653-60.

4. Cutler CS, Lee SJ, Greenberg P, et al. A decision analysis of allogeneic bone marrow transplantation for the myelodysplastic syndromes: delayed transplantation for low-risk myelodysplasia is associated with improved outcome. Blood 2004;104:579-85.

5. Schmid C, Labopin M, Socie G, et al. Outcome of patients with distinct molecular genotypes and cytogenetically normal AML after allogeneic transplantation. Blood 2015;126:2062-9. 